\title{
Quantifying the contribution of intracranial pressure and arterial blood pressure to spontaneous tympanic membrane displacement
}

Authors: Wahbi K. El-Bouri ${ }^{1,2}$, Dario Vignali ${ }^{1}$, Konstantina lliadi ${ }^{1,2}$, Diederik Bulters ${ }^{3,4}$, Robert J. Marchbanks ${ }^{1}$, Anthony A. Birch ${ }^{1,3}$, David M. Simpson ${ }^{2}$

Address:

${ }^{1}$ Neurological Physics Group, Department of Medical Physics, University Hospital Southampton NHS Foundation Trust, Southampton, UK

${ }^{2}$ Institute for Sound and Vibration Research, University of Southampton, Southampton, UK

${ }^{3}$ Faculty of Medicine, University of Southampton, University Hospital Southampton NHS Foundation Trust, Southampton, UK

${ }^{4}$ Wessex Neurological Centre, University Hospital Southampton NHS Foundation Trust, Southampton, UK

Keywords:

Non-Invasive, Humans, Respiratory Rate, Heart Rate, Coherence, TMD, CCFP 


\section{Abstract}

\section{Objective}

Although previous studies have shown associations between patient symptoms/outcomes and the spontaneous tympanic membrane displacement (spTMD) pulse amplitude, the contribution of the underlying intracranial pressure (ICP) signal to the spTMD pulse remains largely unknown. We have assessed the relative contributions of ICP and arterial blood pressure (ABP) on spTMD at different frequencies in order to determine whether SPTMD contains information about the ICP above and beyond that contained in the ABP.

\section{Approach}

Eleven patients, who all had invasive ICP and ABP measurements in-situ, were recruited from our intensive care unit. Their SPTMD was recorded and the power spectral densities of the 3 signals, as well as coherences between the signals, were calculated in the range $0.1-5 \mathrm{~Hz}$. Simple and multiple coherences, coupled with statistical tests using surrogate data, were carried out to quantify the relative contributions of $A B P$ and ICP to sPTMD.

\section{Main results}

Most power of the signals was found to predominate at respiration rate, heart rate, and their harmonics, with little outside of these frequencies. Analysis of the simple coherences found a slight preference for ICP transmission, beyond that from ABP, to the spTMD at lower frequencies (7/11 patients at respiration, $7 / 10$ patients at respiration $1^{\text {st }}$ harmonic) which is reversed at the higher frequencies (2/11 patients at heart rate and its $1^{\text {st }}$ harmonic). Both ICP and ABP were found to independently contribute to the SPTMD. The multiple coherence reinforced that ICP is preferentially being transmitted at respiration and respiration $1^{\text {st }}$ harmonic.

\section{Significance}

Both $\mathrm{ABP}$ and ICP contribute independently to the spTMD signal, with most power occurring at clear physiological frequencies - respiration and harmonics and heart rate and harmonics. There is information shared between the ICP and SPTMD that is not present in ABP. This analysis has indicated that lower frequencies appear to favour ICP as the driver for SPTMD. 


\section{Introduction}

Intracranial pressure (ICP) arises due to cerebrospinal fluid (CSF), brain tissue, and circulating cerebral blood, co-existing within a rigid enclosed space - the skull. The Monroe-Kellie hypothesis thus states that any increase in one of these elements must be compensated by a decrease in another element or a rise in ICP (Mokri, 2001). Abnormal ICP is symptomatic of an underlying pathology such as subarachnoid haemorrhage, stroke, or encephalitis (Czosnyka and Pickard, 2004, Dunn, 2002). Current "gold standard" ICP monitoring methods are highly invasive procedures, requiring access to the ventricles or the brain parenchyma (Kirkman and Smith, 2014, Lundberg, 1960). Due to the invasiveness of the procedure, complications can arise, with the risk of infection (Ghajar, 1995), as well as the potential for haematoma formation, and seizures (Dunn, 2002). Invasive ICP monitoring is only used in conditions where symptoms are severe and there is a need for immediate intervention. Having non-invasive measurements would allow for the screening and diagnosis of patients before symptoms become severe, or for monitoring the natural course and response to treatment of chronic conditions, allowing for better directed intervention to improve outcomes.

As a result of this need, a variety of non-invasive ICP measurement techniques have been developed to help alleviate the difficulties associated with invasive monitoring. These include, but are not limited to, magnetic resonance imaging (Alperin et al., 2000), transcranial Doppler examination (Schoser et al., 1999), and tympanic membrane displacement (TMD) (Marchbanks, 1989). A review of current non-invasive ICP monitoring methods can be found in (Zhang et al., 2017). The TMD technique utilises the direct channel of communication (via the cochlear aqueduct) between the subarachnoid space in the brain and the perilymphatic fluid in the cochlea (Traboulsi and Avan, 2007). This allows ICP changes to be transmitted through the oval window, observed as displacements of the tympanic membrane, and measured as volume changes in the ear canal. These nanolitre changes are detected through the TMD analyzer (Reid et al., 1990, Samuel et al., 1998). There are two forms of TMD measurements: firstly, the evoked measurement, where a $1 \mathrm{kHz}$ sound at $20 \mathrm{~dB}$ above the acoustic reflex threshold is used to activate the stapedial reflex (Phillips and Marchbanks, 1989). Studies have shown a significant correlation between evoked TMD and direct ICP measurements (Gwer et al., 2013, Samuel et al., 1998), although there is large inter-subject variability (Shimbles et al., 2005). Despite this, the method has shown promise to diagnose abnormalities and measure changes within an individual over time (Konradsson et al., 2000, Moss et al., 1991). The second form is the spontaneous measurement, where the TMD analyzer measures the spontaneous volume displacement of the tympanic membrane that arises from the pulsing of ICP and/or arterial blood pressure (ABP) (Gwer et al., 2015, Lehrer et al., 2009).

Gopen et al. (Gopen et al., 1997) calculated that the cochlear aqueduct acts as a low-pass filter transmitting the ICP signal at frequencies below $20 \mathrm{~Hz}$. Thus ICP changes at frequencies as high as $20 \mathrm{~Hz}$ may be expected in the perilymphatic pressure, from where they will be transmitted to the tympanic membrane. It is likely that other pathways, such as pulsations in the outer or middle ear, in addition to ICP transmission through the cochlear aqueduct, contribute to the forces acting on the tympanic membrane. As a result, the spontaneous TMD (spTMD) is assumed to be a superposition of the ICP waveform with the ABP waveform, although venous pressure and respiration may also affect the tympanic membrane displacement. The exact interactions and relative magnitudes of the various physiological signals are not well understood (Finch et al., 2018). A major benefit of spTMD 
would be realised if it is able to indirectly measure the ICP waveform rather than only its mean value; the former may be more important than static ICP in a diagnostic setting (Eide and Kerty, 2011). Gwer et al. (Gwer et al., 2015) demonstrated that increased spTMD pulse measurements correlated with risk of death in children, whilst Lehrer et al. (Lehrer et al., 2009) analysed a number of patient case studies demonstrating a "clear association between symptoms and neuro-otological disorders that cause increased intra-aural pressure waves as recorded by the [spTMD]". Similarly, Shulman et al. (Shulman et al., 2012) found that a smaller spTMD pulse correlated with improved symptoms after treatment of patients with neuro-otological conditions. Despite these studies, the contribution of the underlying ICP signal on the sPTMD waveform remains largely unknown, along with the pathways through which variations in ABP and respiration are transmitted to the spTMD.

Therefore, the aim of this paper is to elucidate the contributions of the ICP and ABP waveforms on the sPTMD signal. A particular challenge exists in separating the contributions of ICP and ABP on the spTMD due to the fact that the ABP affects both the ICP and SpTMD signals - a schematic of the assumed pathways is shown in Fig. 1. Our approach is to determine whether the spTMD waveform contains information about the ICP above and beyond the information contained in the ABP waveform at these frequencies.

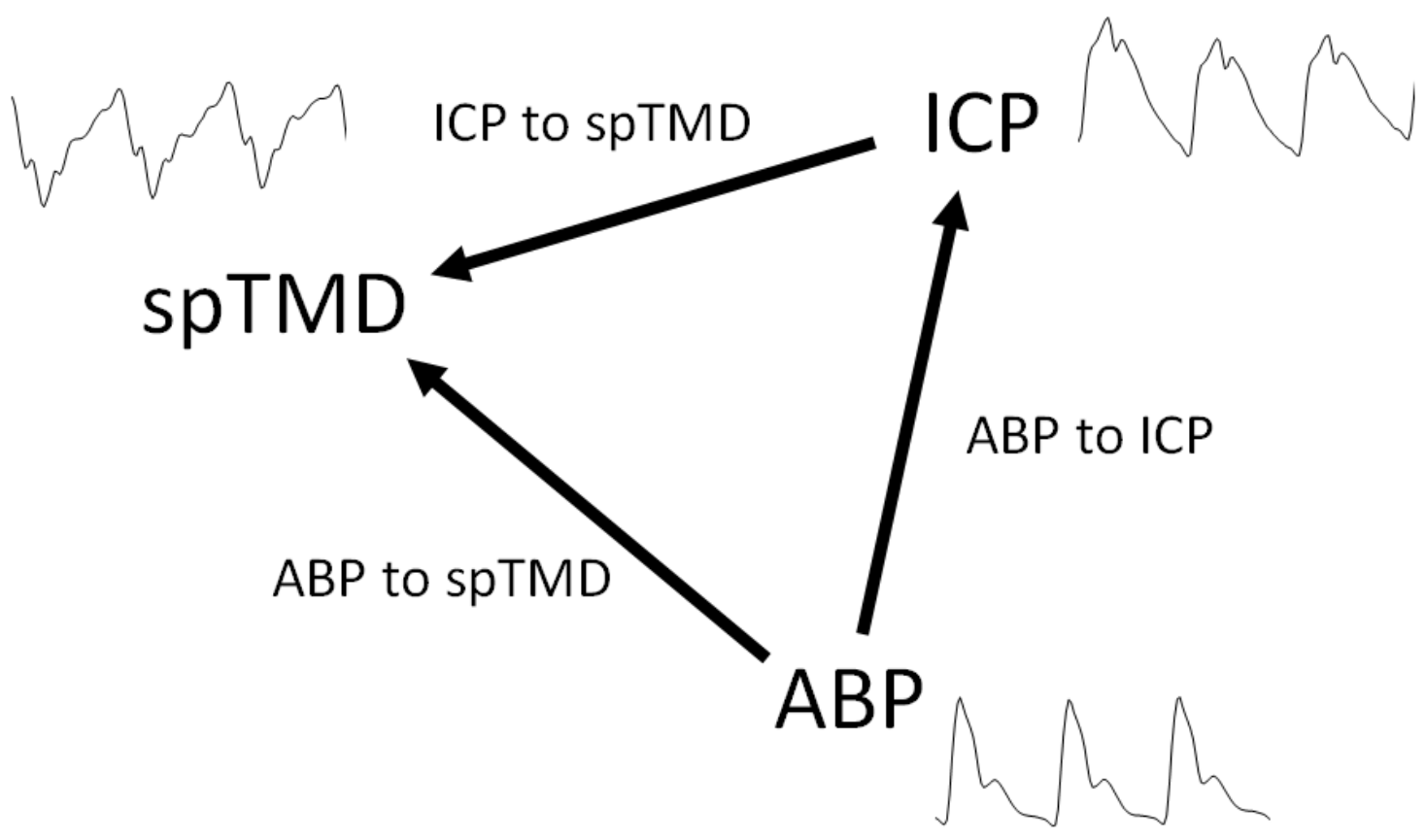

Figure 1 Transmission pathways between the three signals: arterial blood pressure (ABP), intracranial pressure (ICP), and spontaneous tympanic membrane displacement (spTMD). Throughout the paper, the assumed pathways are referred to as 'ABP to ICP', 'ABP to spTMD', and 'ICP to spTMD'. 


\section{Materials and Methods}

\subsection{Patient Population}

The study data was collected under the approval of two National Health Service, Research ethics committees, ref: 14/SC/0301 and 05/Q1704/108. Data was collected over a 22 month period. Patients with invasive monitoring of ICP as part of their clinical management were recruited from our neurosurgical wards or intensive care unit. All patients with invasive ICP monitoring in place were eligible unless they were younger than 4 years old, older than 75 years old or if neither ear could be used due to trauma, wax or otitis media. Data was collected in a single visit to the patient's bedside lasting approximately between 1 and 2 hours. Consent was obtained from the patient or, if the patient lacked capacity, following consultation with a close relative or friend.

\subsection{Data Acquisition}

Otoscopy was carried out in both ears. Further measurements were only performed if the ear was seen to be intact and the ear canal was not occluded by blood or wax. Tympanometry was carried out in both ears using a GSI Tympstar Middle Ear Analyser V2 (Grason Stadler). One ear was then chosen for spTMD monitoring; if a marked asymmetry was seen in the tympanometry results, then the ear with the closest to normal middle ear function was chosen. Otherwise the selection of which ear to use was based on the practicalities of patient position at the time of the measurements. The patients' bed angle and pillow arrangement was not altered for the study. Awake patients were asked to lie still in a comfortable position; unconscious patients were not moved unless a turn was required to give access to the chosen ear. The MMS-14 Cerebral and Cochlear Fluid Pressure (CCFP) analyser (Marchbanks Measurement Systems Ltd., Lymington, UK) was then used to record spontaneous tympanic membrane displacements from the chosen ear. Multiple recordings, typically of 20 - 30 seconds duration, were made with a 4 second interval between them. These duration lengths were chosen as this is a typical maximum length of continuous data that may be collected before the inherent drift in baseline value reaches the limits of the MMS-14 device's dynamic range. If the baseline drift was seen to be smaller than usual, allowing longer duration continuous recordings to be made, then the length of individual recordings was extended up to a maximum of 60 seconds. This quasi continuous data collection (with a periodic 4 second pause to reset the baseline) was continued for as long as was practical before movement of the patient was required. Collected data ranged between 1 hour and 2 hours 15 minutes long, with an average recording of 1 hour 35 minutes.

The MMS-14 analogue output signal was recorded together with the invasive ICP and ABP. Data were sampled at $200 \mathrm{~Hz}$ with proprietary data acquisition software (ICM+, Cambridge Enterprise, Cambridge, UK, http://www.neurosurg.cam.ac.uk/icmplus) and stored for subsequent analysis. The ICP measurement in clinical use at the time was used in the study and these were different for different patients, as follows:- i) an external pressure transducer (Truwave, Edwards Lifesciences, Irvine, US) within the external ventricular drainage system when the drain is closed, ii) A fibreoptic catheter-tip pressure transducer, intraventricular or intraparenchymal, (Integra Camino Plainsboro, US) or iii) A strain gauge catheter-tip pressure transducer, intraventricular or intraparenchymal, (Raumedic, Helmbrechts, Germany.) Arterial blood pressure was measured invasively via a fluid filled catheter in the radial artery (Truwave, Edwards Lifesciences, Irvine, US). All of the invasive pressure transducers used had flat frequency responses across the range of frequencies investigated and a 
low pass filter cut-off that was significantly higher than the $40 \mathrm{~Hz}$ filters used in the monitoring systems, and the maximum frequency of interest. Fluid filled systems were set-up following the manufacturers' guidelines and are therefore not expected to degrade the frequency response characteristics of the pressure monitoring at frequencies lower than $10 \mathrm{~Hz}$. When available the patient's ICP and ABP were recorded as digital signals directly from the patient monitoring system (GE CarescapeB850, GE Healthcare, Chicago, US). In patients who were not being monitored in this way (ward patients) their ICP and ABP were recorded as analogue signals directly from the recording device. All analogue signals were recorded with ICM+ using an A-D converter DT9804-16SE-BNC (Data Translation, Bietigheim-Bissingen, Germany).

\subsection{Data Processing}

The spTMD, ABP, and ICP signals of each of the patients were passed through a $2^{\text {nd }}$ order Butterworth filter with a $20 \mathrm{~Hz}$ cut-off frequency; the signals then being decimated to a frequency of $50 \mathrm{~Hz}$. The frequency response of the MMS-14 CCFP behaves as a low pass filter with a $3 \mathrm{~dB}$ attenuation point at approximately $9 \mathrm{~Hz}$. In the current study, the frequency range of interest is between $0.1-5 \mathrm{~Hz}$ relating to the respiration frequency, heart rate, and their $1^{\text {st }}$ harmonics. The upper frequency limit of $5 \mathrm{~Hz}$ is chosen such that the frequency response is well below the $3 \mathrm{~dB}$ point of the analyser. The limited duration of epochs, together with the drift in the signal, imposes the low-frequency limit of $0.1 \mathrm{~Hz}$ on the analysis. The epochs included in the analysis vary in length from $18-60$ seconds although the majority are between 25 and 30 seconds. The linear trend in the spTMD (mainly due to drift in the signal) was removed from all recorded epochs prior to further analysis, and thus all epochs also had a mean value of zero. The ABP and ICP signals are DC shifted to a zero mean, since only dynamic changes are of interest in this study.

\subsection{Frequency Domain Analysis}

Power spectral densities (PSDs) are used to identify where the majority of power lies in the signals, and hence identify the frequencies of interest. Simple (pair-wise) coherence was used to confirm that spectral peaks in SpTMD are indeed correlated with (and considering the physiology presumably driven by) ABP and ICP oscillations. Multiple coherence was then used to assess the joint and independent contributions of ABP and ICP to SPTMD. The significance of additional contributions of $A B P$ over and above that from ICP (and vice versa) was assessed by surrogate data (Monte Carlo) analysis.

In order to estimate PSD and coherence, overlapped windows 18 seconds in length are chosen; in longer epochs, multiple overlapped 18 second windows are applied. This allows for the same resolution to be used for all patients, giving a spectral resolution of approximately $0.06 \mathrm{~Hz}$ (and hence a lowest frequency of $0.06 \mathrm{~Hz}$ in the analysis), and allows spectral and coherence peaks due to respiration, heart rate, and their harmonics to be clearly identified and compared between patients.

Coherence functions are calculated on the raw signals, between ABP and ICP, ABP and SPTMD, and ICP and SpTMD. It should be noted that these coherence estimates do not take causality between the signals into account and hence do not distinguish between what is an input and an output. Due to device limitations and the resultant uncertainty about the delay between recorded signals, analysis of causal relations (e.g. via Granger causality analysis and directed coherence) was not deemed reliable. A Wilcoxon signed-rank test is used to determine whether there is a significant 
difference in the coherences between the two transmission pathways of interest - ABP to spTMD and ICP to SPTMD. This is done at each relevant frequency - respiration fundamental, respiration $1^{\text {st }}$ harmonic, heart rate fundamental, and heart rate $1^{\text {st }}$ harmonic. Results were deemed significant at $\alpha$ $=0.05$.

Multiple coherence, with ABP and ICP as inputs and spTMD as an output, is also calculated, using the same overlapped windowing as for the simple coherences. In order to determine the significance of these coherence estimates, surrogate data analysis (Monte Carlo simulation) is performed, using random phase for each of the signals (Faes et al., 2004) - in each of the subjects - in turn, with 500 repeats to build up a distribution of coherence values under three different null-hypotheses. Firstly, for the null-hypothesis that SPTMD is not correlated with ICP and ABP, spTMD phase is randomized, its coherence estimated, and this process is repeated 500 times. The resulting distribution is then used to test for statistical significance of the multiple coherence estimates. If the multiple coherence from the recorded signal exceeded the $95^{\text {th }}$ percentile of the null distribution, the multiple coherence estimate is deemed significantly greater than zero. Secondly, for the null hypothesis that $A B P$ does not independently contribute to SPTMD, ABP phase is randomized to give an estimate of the null-distribution of multiple coherence. If the multiple coherence from the recorded signal exceeded the $95^{\text {th }}$ percentile of the null distribution, ABP was deemed to provide a significant additional contribution (beyond that from ICP) to SPTMD. The third hypothesis was that ICP provided no significant additional contribution to SPTMD. To test this, the ICP phase is randomized and the equivalent procedure used for the second hypothesis followed. Of particular interest in this analysis is whether, in different frequency bands, SPTMD should be considered as driven predominantly by $A B P$ or ICP or both signals. The surrogate data analysis method, rather than more conventional statistical approaches, was chosen for two reasons: firstly, the work of Faes et al. (Faes et al., 2004) demonstrated that in signals with narrow-band oscillations, critical values for coherence can vary across the spectrum, whereas conventional estimators of the critical values give these as being constant e.g. (Benignus, 1969). Secondly, the estimates are made from overlapping and nonoverlapping windows (depending on the length of epochs included), which would make it very difficult to estimate the correct degrees of freedom needed in finding critical values by conventional approaches.

\section{Results}

\subsection{Patient Population}

Eleven patients were recruited, 8 male and 3 female, with a mean age of 45 (range $17-73$ ). Characteristics of each patient are shown in Table 1. Ten of the eleven patients were ventilated and sedated. Both parenchymal and extra ventricular drain (EVD) transducers were used to measure the ICP; EVD's were always clamped while ICP was being measured. The average middle ear pressure was $-55 \mathrm{daPa}$, with a range of $-135-37.5 \mathrm{daPa}$. 


\begin{tabular}{llllll}
\hline Patient & Condition & $\begin{array}{l}\text { Mean ICP } \\
(\mathbf{m m H g})\end{array}$ & ICP Transducer & $\begin{array}{l}\text { Mean ABP } \\
(\mathbf{m m H g})\end{array}$ & Ventilated \\
\hline $\mathbf{1}$ & TBI & 16 & Parenchymal & 84 & YES \\
$\mathbf{2}$ & Tumour & 6 & EVD & 102 & YES \\
$\mathbf{3}$ & TBI & 12 & Parenchymal & 98 & YES \\
$\mathbf{4}$ & TBI & 18 & EVD & 90 & YES \\
$\mathbf{5}$ & TBI & 13 & Parenchymal & 90 & YES \\
$\mathbf{6}$ & TBI & 16 & Parenchymal & 90 & YES \\
$\mathbf{7}$ & TBI & 12 & Parenchymal & 86 & YES \\
$\mathbf{8}$ & TBI & 16 & EVD & 88 & YES \\
$\mathbf{9}$ & Infection & 9 & EVD & 90 & YES \\
$\mathbf{1 0}$ & SAH & 15 & EVD & 100 & NO \\
$\mathbf{1 1}$ & SAH & 6 & EVD & 95 & YES \\
\hline
\end{tabular}

Table 1 Characteristics of the 11 patients used in this study. TBI - traumatic brain injury, SAH subarachnoid haemorrhage, EVD - external ventricular drain.

\subsection{Patient Data}

Two examples of the recordings are given in Fig. 2. Via visual inspection it is evident that the heart rate appears in both the ICP and SpTMD signals. In addition to this, the respiratory frequency also appears to be modulating the three signals (especially apparent in patient 5). As expected, the morphology of ABP is similar in all patients, but that of ICP and spTMD differs quite markedly.
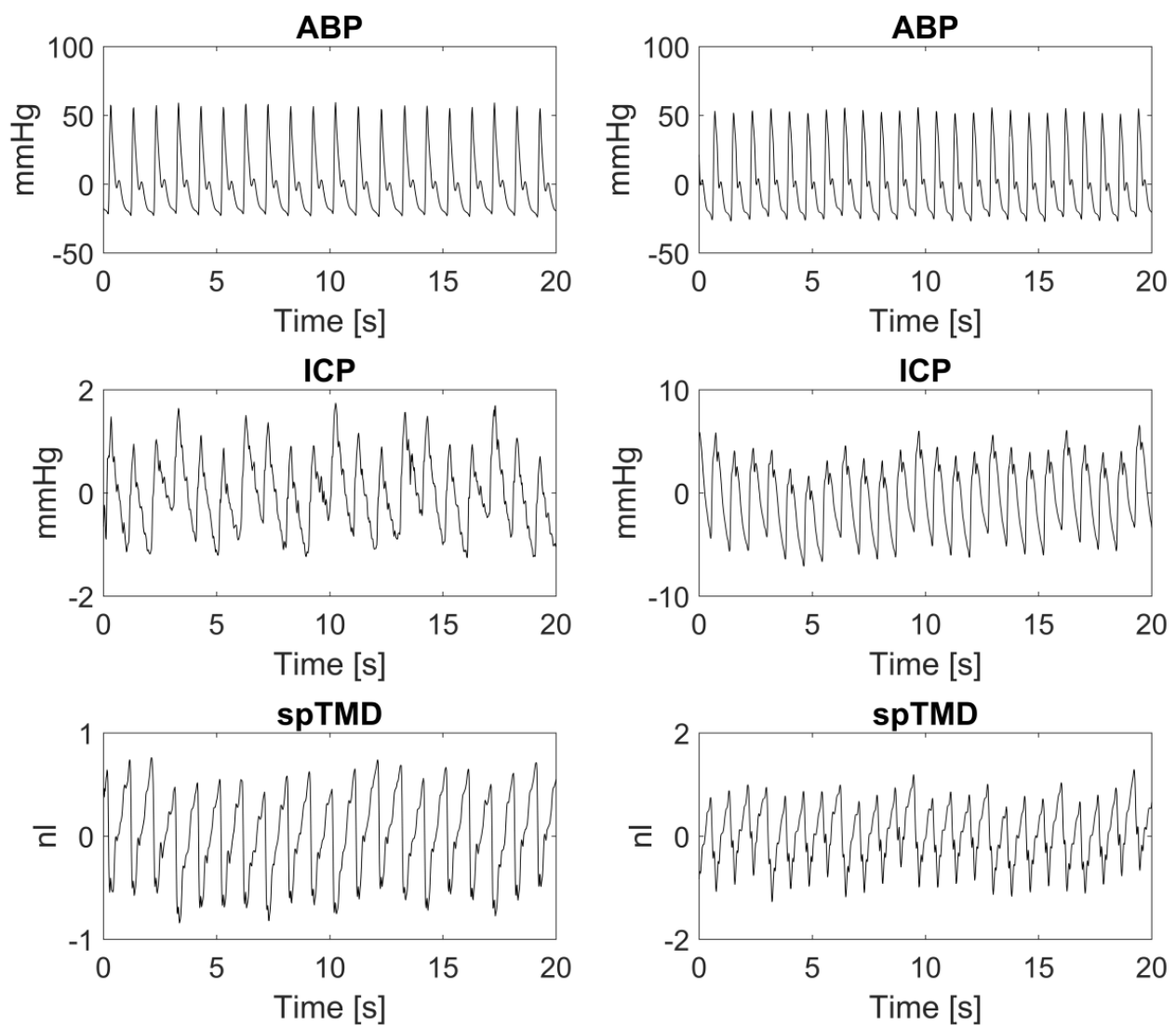

Figure 2 Examples of spontaneous tympanic membrane displacement (spTMD), intracranial pressure (ICP) and arterial blood pressure (ABP) in patient 2 (left column) and in patient 5 (right column). The data has been detrended and shifted to a zero mean. 
The power spectral densities of patient 5 are shown in Fig. 3. As can be seen, the vast majority of power in the signals lies at the frequencies of respiration and heart rate and their harmonics, with little power between these frequencies, except at the very low frequencies $(0.06-0.12 \mathrm{~Hz})$. This is typical of the spectra observed in all signals.

It should be noted that although the $2^{\text {nd }}$ harmonic of respiration was also present in some patients, this often coincided with the heart rate and so was indistinguishable in the analysis. As a result, only the fundamental and $1^{\text {st }}$ harmonic of respiration, and the heart rate and its $1^{\text {st }}$ harmonic were chosen for more detailed analysis. All analysis was undertaken at these individual physiologically chosen frequencies, as opposed to a set of fixed frequencies, due to the variation of respiration rate and heart rate between patients.

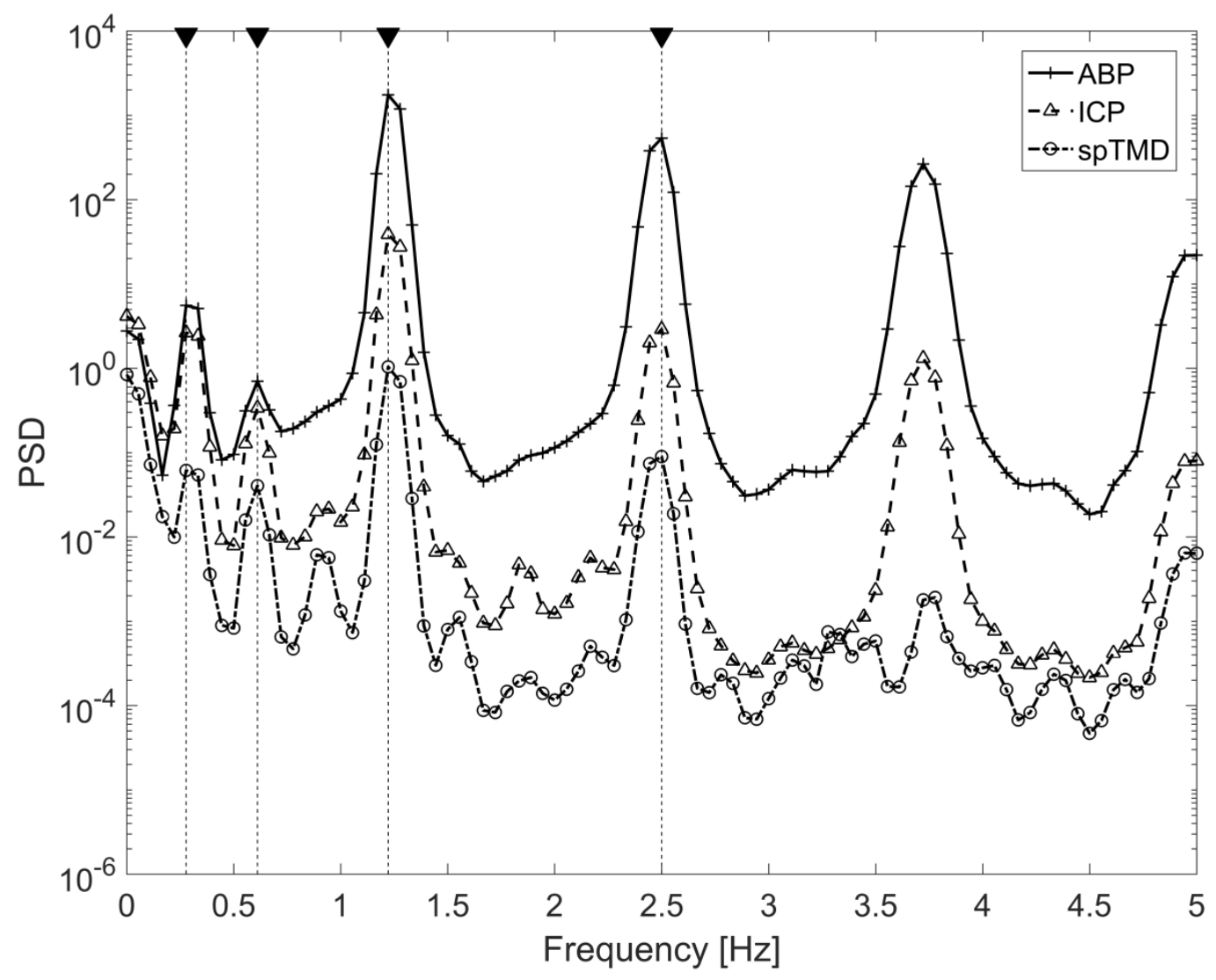

Figure 3 Example power spectral densities (PSD) for patient 5. The PSDs for ABP, ICP, and SPTMD are plotted. The 4 frequencies of interest are determined (vertical dotted lines with arrows). They are, left to right, respiration; respiration $1^{\text {st }}$ harmonic; heart rate; heart rate $1^{\text {st }}$ harmonic. The PSD spectra are in arbitrary units to facilitate comparison.

\subsection{Simple Coherence}

The coherence (magnitude squared) plots can be seen for 2 typical patients in Fig. 4 . There is clear coherence between the pairs of signals at the respiration rate, heart rate, and their harmonics, with much less coherence between these frequencies. Despite the high power at the low frequencies $(0.06-0.12 \mathrm{~Hz})$ there appears to be very little coherence at these low frequencies. 
Higher harmonics of respiration can also be identified in both patients in Fig. 4. This is only generally the case when respiration harmonics frequencies do not coincide with heart rate and its harmonics. It should be noted that the respiration rate coherence peaks tend to be sharp due to the use of ventilators in 10 out of the 11 patients.
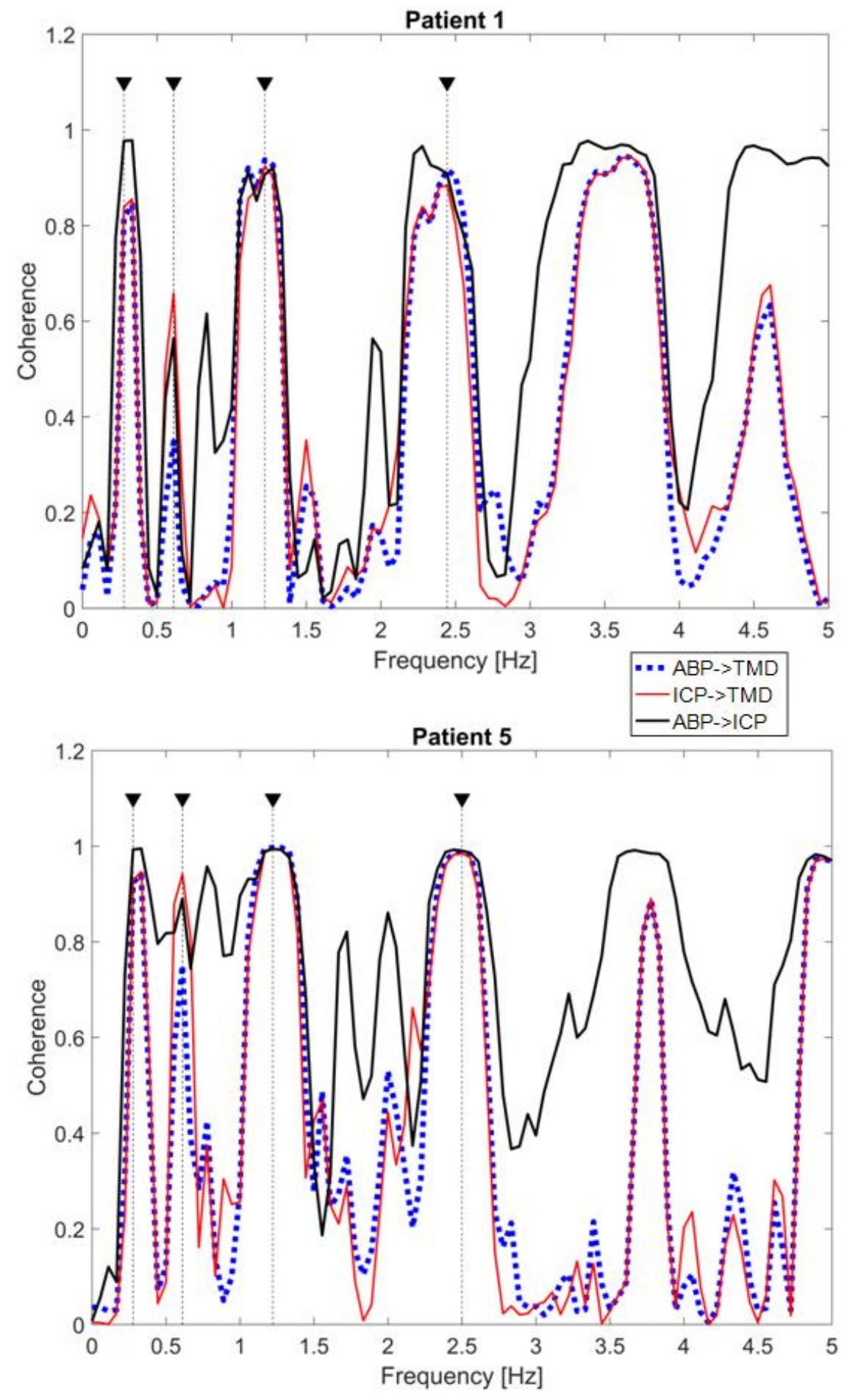

Figure 4 Examples of the coherence (magnitude squared) functions obtained between $A B P$ and spTMD, ABP and ICP, and ICP and SPTMD, along with the arrows identifying the respiration rate and its harmonic, and the heart rate and its harmonic. These are plotted for patients 1 and 5. 
In general, the coherence between $\mathrm{ABP}$ and ICP is greater than for ABP and SPTMD, and ICP and spTMD. Of more interest in the current work are the coherences between ABP and SPTMD and ICP and SPTMD. On the whole, there is very little difference between these two coherences, with the shapes of the coherence plots being very similar (Fig. 4). The mean, median, min and max values of coherence across the 11 patients are also shown in Table 2.

\begin{tabular}{|c|c|c|c|c|c|}
\hline \multirow{2}{*}{$\begin{array}{l}\text { Physiological } \\
\text { Frequency }\end{array}$} & & \multicolumn{4}{|c|}{ Coherence } \\
\hline & & $A B P$ to $s p T M D$ & $A B P$ to $I C P$ & ICP to spTMD & $\begin{array}{c}\text { Multiple } \\
\text { Coherence }\end{array}$ \\
\hline \multirow{4}{*}{ Respiration } & Mean & 0.691 & 0.895 & 0.663 & 0.778 \\
\hline & Median & 0.714 & 0.968 & 0.714 & 0.826 \\
\hline & Min & 0.1118 & 0.5 & 0.189 & 0.462 \\
\hline & Max & 0.985 & 0.993 & 0.993 & 0.993 \\
\hline \multirow{4}{*}{$\begin{array}{l}\text { Respiration } 1^{\text {st }} \\
\text { Harmonic }\end{array}$} & Mean & 0.355 & 0.65 & 0.505 & 0.598 \\
\hline & Median & 0.373 & 0.702 & 0.448 & 0.519 \\
\hline & Min & 0.033 & 0.225 & 0.016 & 0.06 \\
\hline & Max & 0.751 & 0.894 & 0.961 & 0.978 \\
\hline \multirow[t]{4}{*}{ Heart Rate } & Mean & 0.924 & 0.935 & 0.896 & 0.943 \\
\hline & Median & 0.984 & 0.98 & 0.949 & 0.987 \\
\hline & Min & 0.466 & 0.769 & 0.511 & 0.614 \\
\hline & Max & 0.998 & 0.994 & 0.994 & 0.998 \\
\hline \multirow{4}{*}{$\begin{array}{l}\text { Heart Rate } 1^{\text {st }} \\
\text { Harmonic }\end{array}$} & Mean & 0.851 & 0.909 & 0.807 & 0.872 \\
\hline & Median & 0.928 & 0.958 & 0.884 & 0.941 \\
\hline & Min & 0.084 & 0.641 & 0.113 & 0.165 \\
\hline & Max & 0.995 & 0.991 & 0.985 & 0.995 \\
\hline
\end{tabular}

Table 2 The mean, median, maximum, and minimum values of the pair-wise coherences (ABP to spTMD, ABP to ICP, and ICP to SpTMD) and the multiple coherence across the four physiological frequencies of interest. Values are derived from 11 patients except for the respiration $1^{\text {st }}$ harmonic where only 10 patients displayed a prominent peak.

In order to show the relative strength of coherences at the 4 physiologically chosen frequencies for all patients, Fig. 5 displays the difference in the pair-wise coherence for each patient. The difference in coherence is defined as the ICP to spTMD coherence minus the ABP to SPTMD coherence - hence a positive difference indicates a stronger link for ICP to SPTMD transmission and a negative difference indicates a stronger link for ABP to spTMD transmission. Given the individual differences in respiration frequency and heart rate, the grand-average coherence across the 11 patients would not be very meaningful, and so analysis at these four physiologically significant frequencies was deemed more informative. 


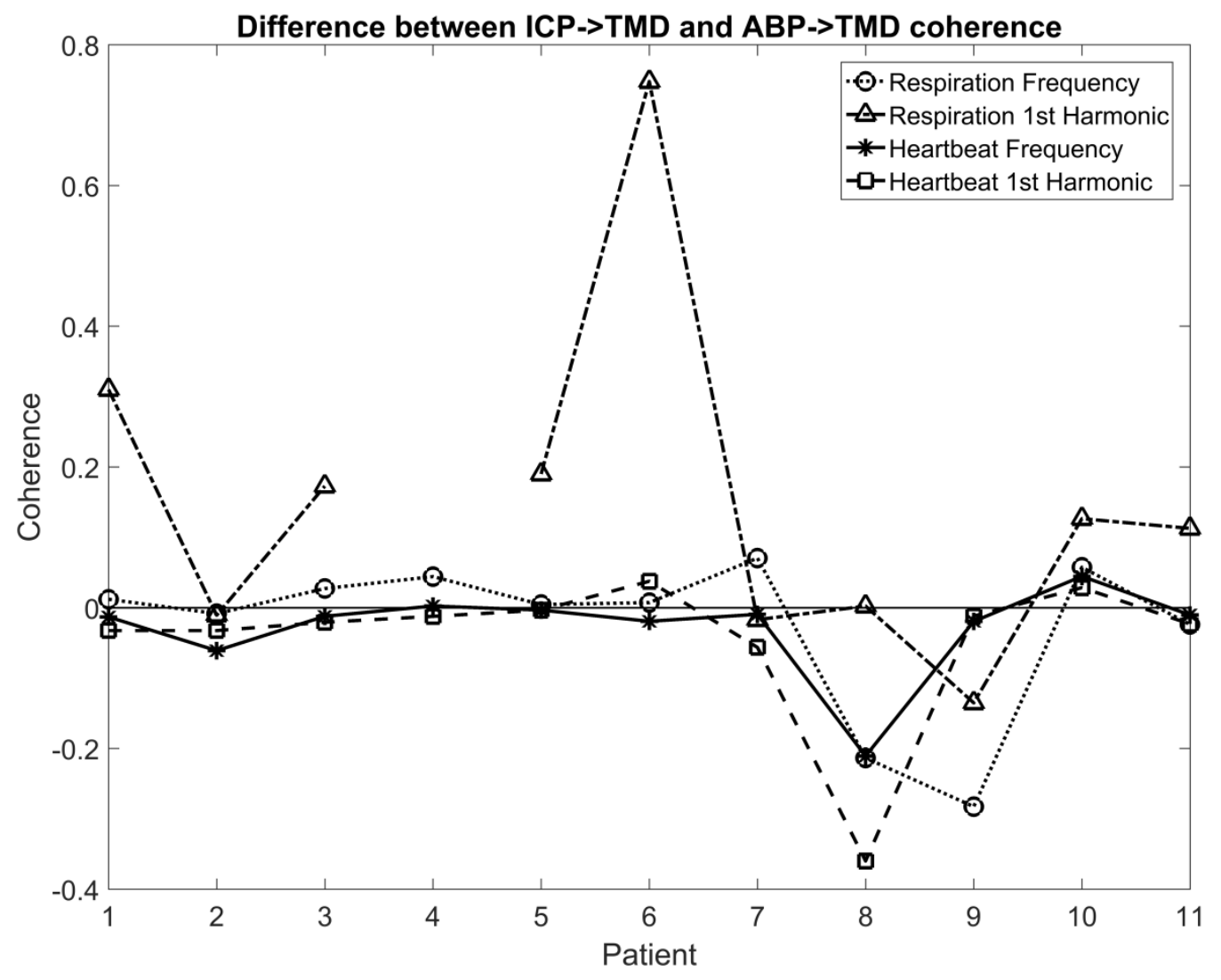

Figure 5 The difference in coherence between ICP to spTMD and ABP to spTMD at the 4 frequencies of interest for the 11 patients. The $1^{\text {st }}$ harmonic of respiration was indiscernible in patient 4 and hence not plotted.

Statistical analysis (Wilcoxon rank-sum tests) comparing the two coherences are given in Table 3, together with the number of cases in which this trend was followed.

\begin{tabular}{lll}
\hline & $\begin{array}{l}\text { Larger coherence and fraction } \\
\text { of cases that followed that } \\
\text { trend }\end{array}$ & $\mathbf{p}$ \\
\hline Respiration & ICP to spTMD (7/11) & 0.76 \\
Respiration $\mathbf{1}^{\text {st }}$ Harmonic & ICP to spTMD (7/10) & 0.11 \\
Heart Rate & ABP to spTMD (9/11) & $\mathbf{0 . 0 4 2}$ \\
Heart Rate $\mathbf{1}^{\text {st }}$ Harmonic & ABP to spTMD (9/11) & 0.12 \\
\hline
\end{tabular}

Table 3 The $p$-value ( $p$ ) for the difference between the two transmission methods (ABP to spTMD and ICP to spTMD) at each frequency of interest. Wilcoxon signed-rank test, $\alpha=0.05$

\subsection{Multiple Coherence}

The results of the multiple coherence for patient 3 (as an illustrative example) can be seen in Fig. 6 . The circles indicate the frequencies at which the coherence is not significant. For example, the circle at the peak in Fig. 6b) at respiration frequency (left-most arrow) indicates that the multiple coherence at this frequency is not significantly larger than with random ABP. However, in Fig. 6c) the lack of a circle indicates the multiple coherence is significantly larger than with random ICP. This indicates that ICP is providing a significant contribution to spTMD at this frequency, over and above 
that from $A B P$, and hence spTMD at the respiration frequency contains more information about the ICP than the ABP. As would be expected the randomised spTMD signal gives a very low critical coherence indicating that at almost all frequencies $A B P$ and ICP together contribute significantly to the spTMD signal.

a)

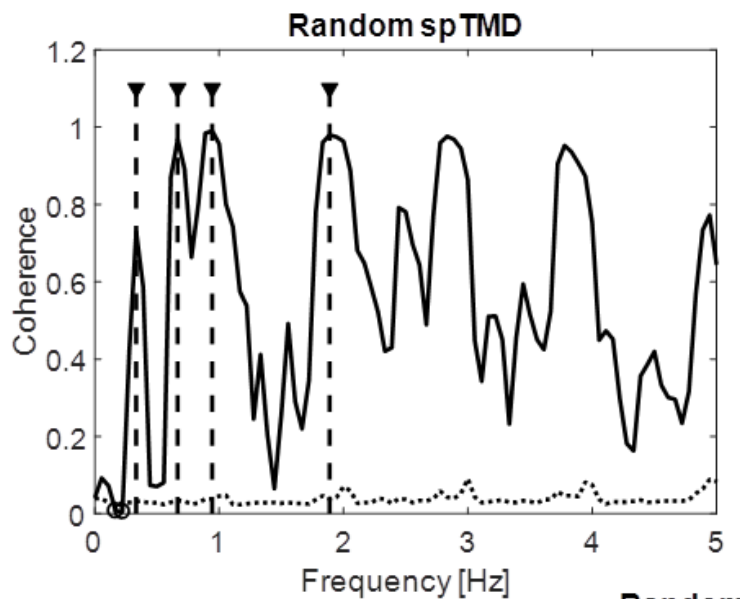

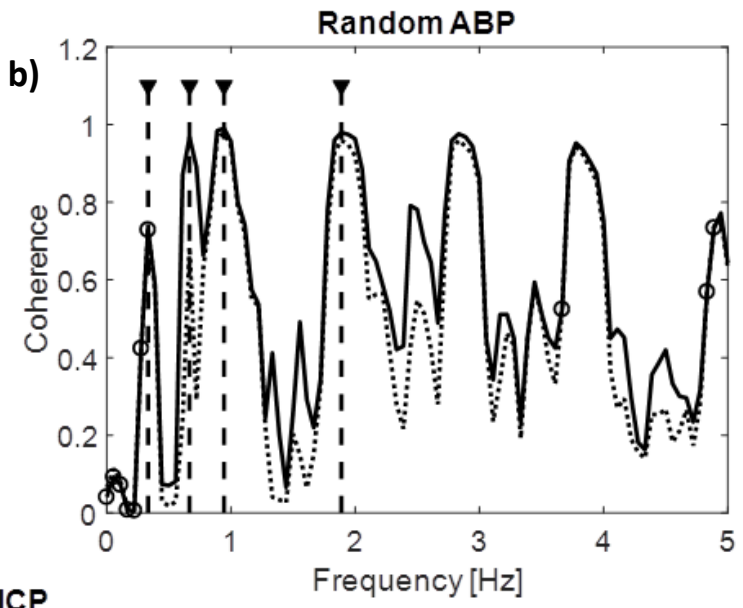

c)

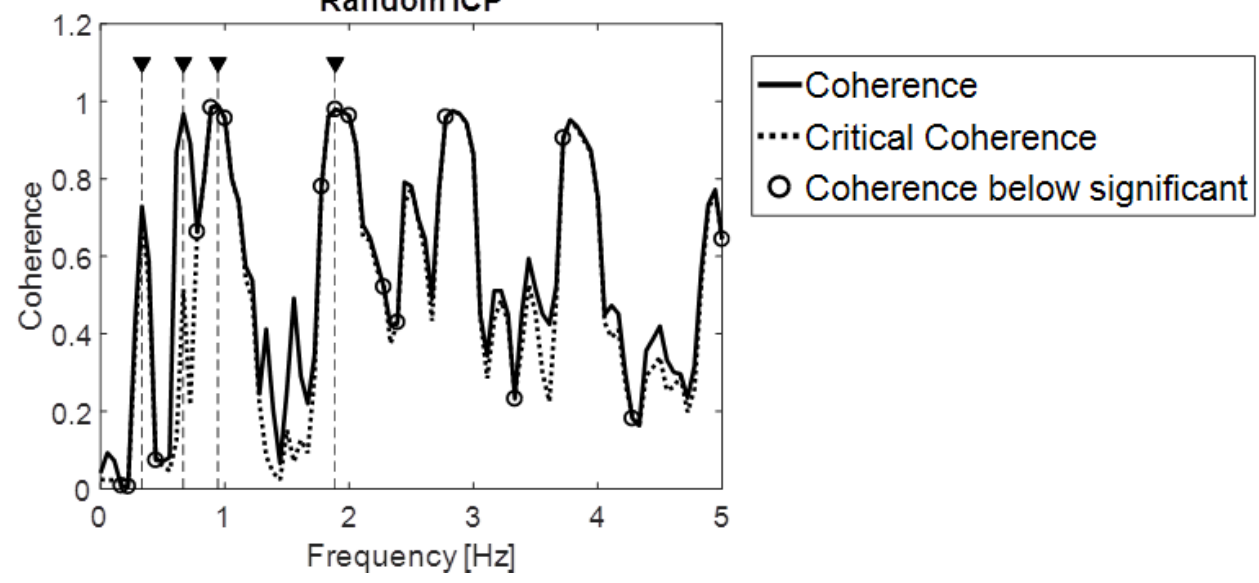

Figure 6 Multiple coherence estimates for patient 3 (solid lines, identical for the three plots). The dotted lines are the $95 \%$ critical coherence generated by 500 repeats of the randomized phase signal for: a) SPTMD, b) ABP, and c) ICP. A plotted circle indicates that the measured signal does not significantly add to the multiple coherence at that frequency. The absence of a plotted circle indicates a significant contribution of that signal (beyond that achieved by spectrally equivalent random signals) to the multiple coherence.

Most frequencies have significant multiple coherence for both phase randomised $A B P$ and ICP, i.e. both ICP and ABP make (independent) contributions to spTMD. The respiration $1^{\text {st }}$ harmonic (second arrow from the left) is one such example in Fig. 6. Inspecting the critical coherence (the dotted line in Fig. 6 - defined as the $95^{\text {th }}$ percentile of the distribution of coherence values, from 500 simulations, for the phase randomised signal) at this frequency, however, shows that the coherence for ICP is further from its critical value than that of $A B P$, suggesting that the former is more highly significant, though both are significant contributors to spTMD. The difference between these critical coherences is plotted in Fig. 7. As can be seen, at the respiration and respiration $1^{\text {st }}$ harmonic frequencies, there is a positive difference in the critical coherence (hence a smaller critical ICP coherence) and so ICP provides more information to the spTMD at this frequency. At the heart rate 
and heart rate $1^{\text {st }}$ harmonic, variations in SPTMD appear to be driven predominantly by $A B P$, although ICP also explains a large part of the coherence.

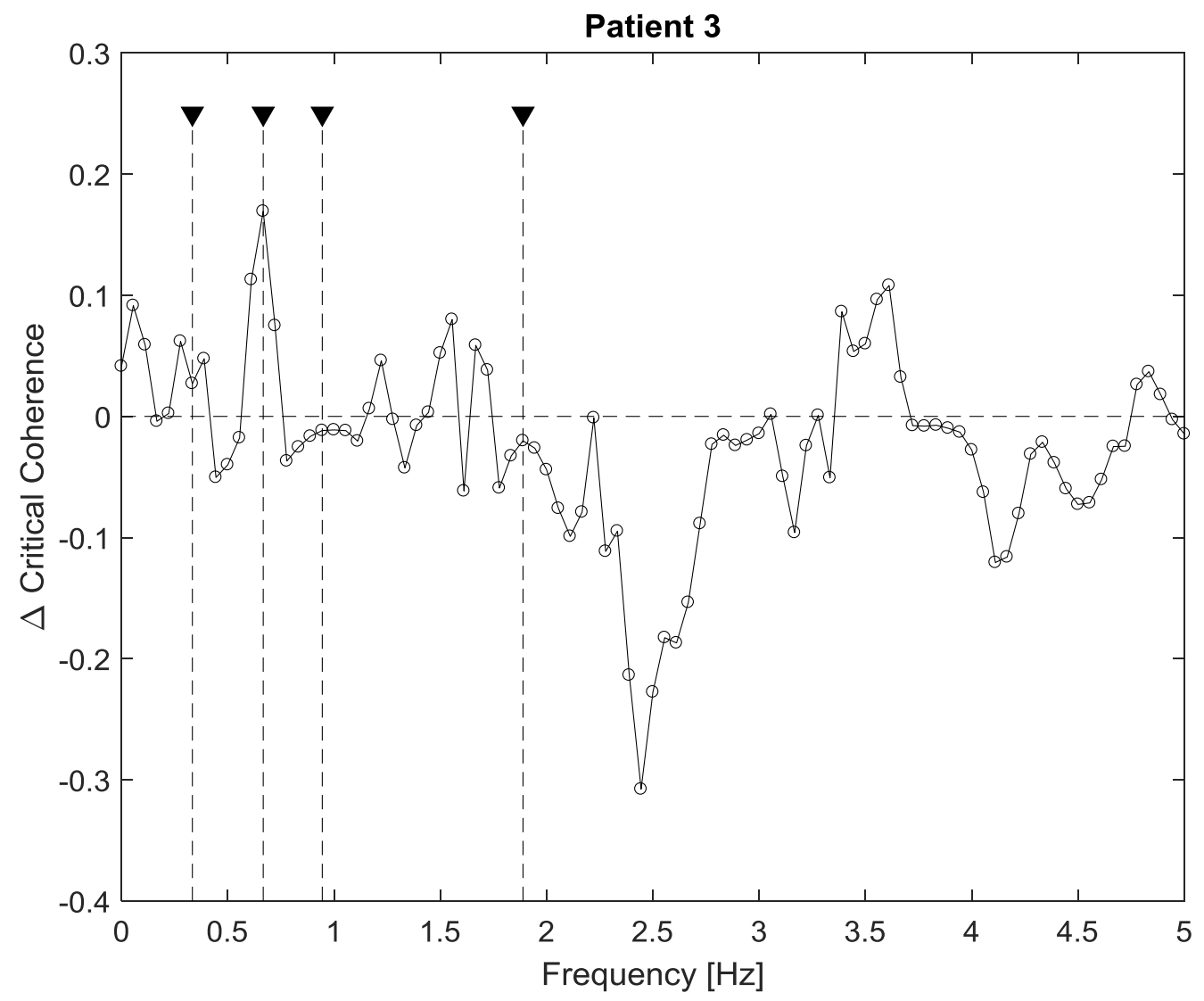

Figure 7 Difference in the critical coherences - the dotted line in Fig. 6b) minus the dotted line in Fig. $6 c$ ) - between phase shuffled ABP and phase shuffled ICP for patient 3. A positive value in this difference indicates a stronger multiple coherence with the ICP signal than with the ABP signal.

The difference in the critical coherences - along with the median - is plotted in Fig. 8 for every patient. These are plotted at each frequency of interest. Both the respiration and respiration $1^{\text {st }}$ harmonic have medians above zero, whilst the heart rate and its harmonic have medians below zero. A statistically significant (Wilcoxon rank sum test) non-zero median was found for the respiration $1^{\text {st }}$ harmonic (median $=0.10, p=0.049$ ) and for the heart rate (median $=-0.01, p=0.042$ ). It is also apparent that the differences in coherence for most patients are small, as indicated by the clustering of results close to the zero difference line (Fig. 8) - which together with the high coherence values indicates the very large joint contribution made to spTMD by both ICP and ABP. 


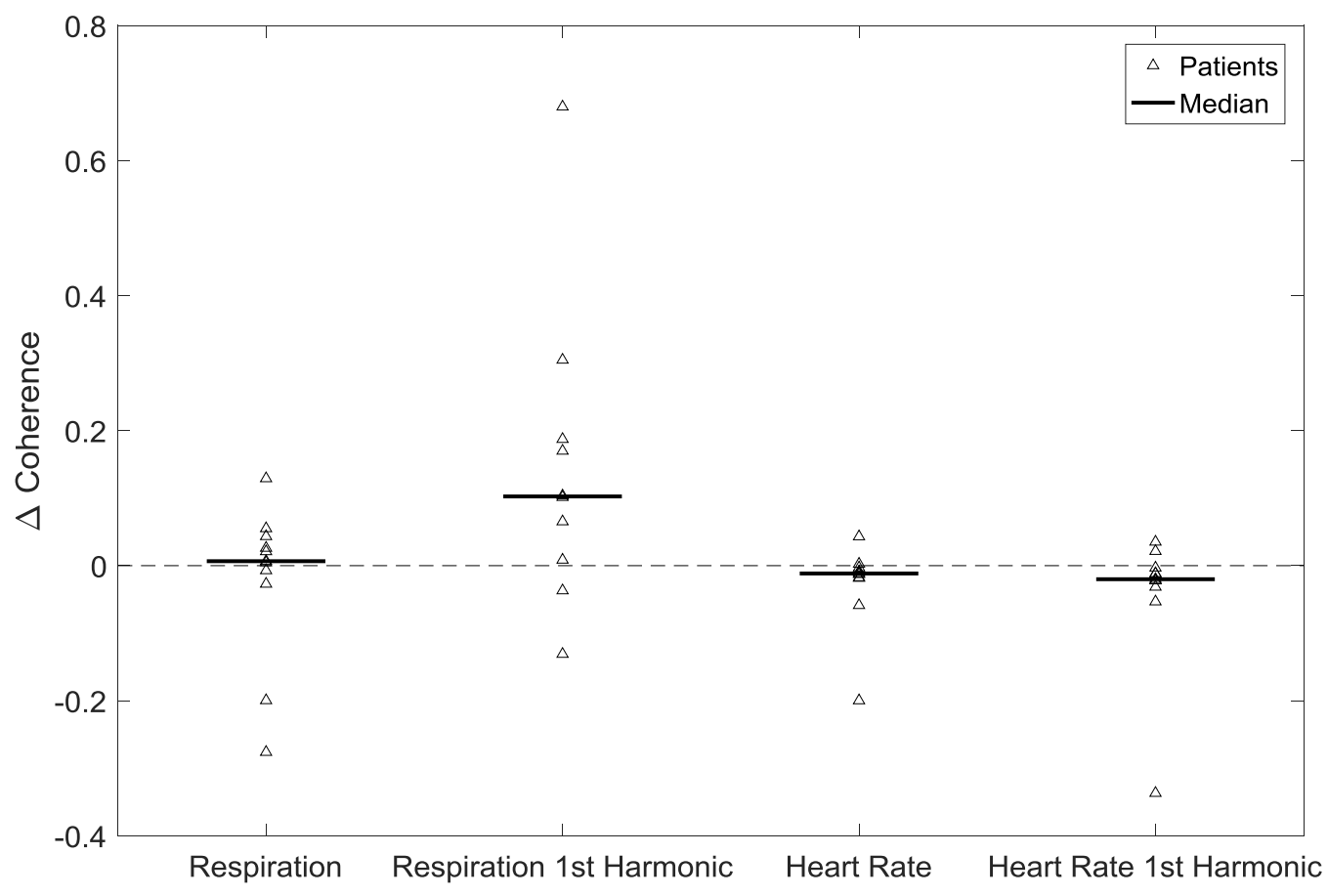

Figure 8 The difference in the critical coherences for each patient at each frequency, along with the median value (horizontal bar). A positive value indicates a greater coherence between the ICP and spTMD as opposed to the ABP and spTMD.

It should be noted here that patient 3 (Fig. 6) is an example where the lowest frequencies (0.06- 0.12 $\mathrm{Hz}$ ) also have significant multiple coherence, albeit much lower than those at the spectral peaks. Interestingly, the multiple coherence is significant with randomised ICP, but not with randomised $A B P$, indicating that these low frequencies are potentially transmitted from ICP to spTMD. However, this is not the case for all patients: only $4 / 11$ of the patients having significant coherence at frequencies below the respiratory peak. This does indicate that there may be preferential ICP transmission at these low frequencies also, although the limited recording time of the SPTMD and the potential for low frequency noise due to the drift in the spTMD recording obfuscates this observation and does not allow robust generalization.

\section{Discussion}

Prior to this study the source of SPTMD variations had received little attention beyond rather speculative suggestions. In this paper we have quantified the contributions of ICP and ABP to the spTMD signal for the first time, and have shown that both ICP and ABP contribute independently to the spTMD signal. As well as this, the majority of power in SPTMD, in the frequency band studied, has been shown to lie at clear physiological frequencies (respiration and its harmonics and heart rate and its harmonics), with only much lower coherences (and not always statistically significant) at frequencies below respiration, down to the lowest frequencies resolvable $(\approx 0.06 \mathrm{~Hz})$. Analysis of the simple coherences between the 2 transmission pathways of interest (ABP to spTMD and ICP to spTMD) indicated strong coherences at the above physiological frequencies with little coherence 
between them, and little significant coherence at the lowest frequencies; again indicating that most information lies at the four physiological frequencies chosen. It is likely that low frequency noise (including the drift) is contributing to the power observed at the lowest frequencies. Therefore, whilst the lowest frequencies are of great interest in the study of the dynamics of ICP and hence in spTMD, it was not possible to analyse these with the current data set.

At respiration and respiration $1^{\text {st }}$ harmonic, there was a preference for the ICP to SpTMD pathway with $7 / 11$ and $7 / 10$ patients having larger coherences at those frequencies, whereas only $2 / 11$ patients had a higher ICP to SPTMD coherence at heart rate and its $1^{\text {st }}$ harmonic. This indicates that ICP is the stronger component at these lower frequencies (although the difference in coherence was not significant), whilst at higher frequencies ( $>1 \mathrm{~Hz}$ ) ABP has the greater influence on the spTMD signal (significance was demonstrated for the heart rate frequency). This is of interest as it points towards the analysis of lower frequencies in the SpTMD signal as having less contamination from the $A B P$ allowing better potential for extraction of the ICP contributions, and potentially assessment of ICP non-invasively. In particular, the spTMD measurements could relate to B-waves or slow waves in the low frequency ICP dynamics. Autoregulation is generally considered to be most effective at very low frequencies (Czosnyka et al., 2009, Panerai, 2008), and well below the frequencies of the oscillations considered in this paper. The preferential transmission of ICP at lower frequencies can be explained by considering the cochlear aqueduct as a low-pass filter (Gopen et al., 1997). Gopen et al. determined the cut-off of the cochlear aqueduct as being $20 \mathrm{~Hz}$ for a fully patent aqueduct, with an average diameter and length. However, it is a possibility that the large natural variability in sizes and patency of the aqueducts can lead to much smaller frequency cut-offs, and hence only low frequency transmission through the cochlear aqueduct, whilst filtering out the heart pulse frequencies.

The simple coherences were corroborated by the multiple coherence analysis, which again demonstrated that at lower frequencies the ICP signal had a greater coherence with spTMD than the ABP signal $-7 / 11$ and $8 / 10$ patients for the respiration rate and its harmonic. This was reversed at the heart rate and its harmonic (both $2 / 11$ patients). When comparing the difference in critical coherences between phase shuffling the ICP and ABP (Fig. 8) both the respiration rate and its harmonic had a positive median (indicating ICP made a greater contribution), whilst the heart rate and its harmonic had a negative median (indicating more information is contained in the ABP). The respiration $1^{\text {st }}$ harmonic and heart rate were significantly non-zero, which again points towards the analysis of lower frequencies to elucidate the ICP contribution to the spTMD. The sample available clearly indicates some inconsistency between individuals and at best trends across the cohort. A larger sample may provide more significant results in Table 3, but would not resolve the diversity in individual responses.

The assumed mechanisms of transmission from the ICP to the spTMD and the ABP to the SPTMD are not straightforward. The main transmission path from ICP to the tympanic membrane is assumed to be via the cochlear aqueduct which behaves like a low pass filter transmitting the pressure waves to the perilymphatic fluid (Gopen et al., 1997). The perilymphatic fluid then applies a pressure to the stapes footplate which transmits the pulsatility across the ossicular chain and displaces the tympanic membrane (Marchbanks, 1996). This pathway is not present in those who have an occluded cochlear aqueduct (Carlborg et al., 1982). The status of the cochlear aqueduct was unknown in the patients we tested. Studies using postural changes and evoked TMD indicated at least $81 \%$ of normal healthy 
subjects of this age range have a patent cochlear aqueduct in at least one ear and that for an individual ear, between $63 \%$ and $71 \%$ of cochlear aqueducts are patent (Campbell-Bell et al., 2017). This would imply that between 3 and 4 out of 11 of our patients may have had occluded cochlear aqueducts in the ear tested. The ABP pulsatility is transmitted to the ICP via the variations in arterial blood volumes in the brain, transmitting the pressure through the brain parenchyma and CSF (Wagshul et al., 2011). The direct route from ABP to spTMD is likely due to the vascularisation of the outer and middle ear, resulting in a pulsatility that either affects the volume of the ear canal, or is directly transferred to the tympanic membrane. The inner ear will also be vascularised and hence some of the ABP pulsations may be transmitted via the perilymphatic fluid (Finch et al., 2018). The vascular supply to and drainage from the cochlea are both from an intracranial route and are therefore themselves a combination of intracranial and arterial pressure waveforms. Due to the convoluted transmission pathways, it is likely that the measured ICP and ABP waveforms used in this study are modified prior to transmission at the tympanic membrane. However, it is likely such modifications will affect the higher frequencies far more than the lower.

Only the ABP to spTMD and ICP to spTMD pathways were considered in this paper. There will, however, be other pathways and physiological signals affecting the spTMD. In particular, venous pressures in the ear are likely to be the largest contributor that has not been analysed in this paper. Central venous pressure (CVP) can be measured and may be representative of the extracranial venous pressures in the ear. In lieu of any such recorded data, the analysis was conducted with only the ABP and ICP signals. In future studies we propose to collect CVP along with the other signals; this may then also be used as a third input to help clarify its contribution to the spTMD. Another potential pathway is the airway pathway through the Eustachian tube to the middle ear causing changes in pressure. If the Eustachian tube opens during a recording this can be expected to have a profound and dominating impact on the spTMD waveform. However, for the majority of the time, and particularly in paralysed ventilated patients, this is likely closed and hence not affecting the mechanics of the middle ear. Despite this, the average middle pressure for the patients was -55 $\mathrm{daPa}$, which is outside the normal range of -50 to $50 \mathrm{daPa}$. Such low values are expected in patients who have been artificially ventilated. The below average middle ear pressures may have an effect on the magnitude of the SPTMD, although it is not clear that this is likely to alter the relative contributions of ABP and ICP to the TMD waveforms analysed in this study.

It should be noted that the coherence analysis presented here is a linear technique. Therefore, it will not be able to pick up potential non-linear physiological affects between the signals. Considering the high levels of coherence observed the linear model appears to provide an acceptable first approximation. It should also be pointed out that with strongly periodic signals, the frequency binwidth in coherence estimation can have a profound effect on the amplitude of peaks in coherence. When bins are narrower they will contain less broadband noise while still including most of the narrow-band signal power, thus increasing signal-to-noise ratio in each frequency bin and hence increasing coherence.

Finally, our lower frequency range was limited due to the drift in the MMS-14 CCFP analyser, which meant the spTMD measurement had to be reset every 20 - 30 seconds. This limited the lower frequency range to approximately $0.06 \mathrm{~Hz}$. As a result, low frequencies and static ICP could not be analysed. The focus of this work was chosen to be on the higher frequencies; follow on work will consider lower frequencies, but given the nature of the recorded signals (epochs with drift), will 
require a quite distinct approach. A low frequency analysis requires long duration spTMD signals, which are reliant on a perfect seal and constant air and ear canal temperature. These conditions are extremely difficult to achieve; however, an alternative approach is to extract an spTMD pulse amplitude signal from the raw spTMD signal. This would generate a quasi-continuous SPTMD pulse amplitude signal that is robust across the epochs and affected little by the inherent drift in the system, hence allowing investigation of lower frequencies than currently possible. This is of particular interest, as this paper has indicated that lower frequencies contain more information about the ICP than the ABP. Furthermore, low frequency intracranial pressure waves are known to exist and these provide extremely important information on the condition and pathophysiology of the brain (Momjian et al., 2004, Spiegelberg et al., 2016).

\section{Conclusions}

The aim of this paper was two-fold: firstly, to determine whether ICP and ABP are reflected in the spTMD signal; secondly, to quantify whether the SpTMD signal contained information about the ICP above and beyond that found in the ABP. It was found that both the ICP and ABP waveforms are transmitted to the spTMD, with both independently contributing to the spTMD. Both signals had a very large coherence with the spTMD waveform, and importantly also with one another with little to differentiate between them. As a result, we cannot fully separate the relative contributions of $A B P$ and ICP. However, the analysis provided a tantalizing suggestion that at lower frequencies $(<1 \mathrm{~Hz}$ and in particular at the $1^{\text {st }}$ harmonic of respiration) the SpTMD tended to contain more information about the ICP. At higher frequencies, $(>1 \mathrm{~Hz})$ it appeared that ABP dominated the spTMD signal. As a result, it is suggested that future investigation of the spTMD should concentrate on low frequencies in order to disentangle the ICP contribution, with the suggestion from our results that at even lower frequencies than those analysed in this paper $(<0.1 \mathrm{~Hz})$ ICP will be even more clearly transmitted to spTMD.

\section{Acknowledgements}

We would like to acknowledge the very helpful support from clinical staff in the Neurological Intensive Care Unit of the Wessex Neurological Centre where the measurements were performed.

\section{Conflict of Interest}

Dr. Robert Marchbanks is the Managing Director of Marchbanks Measurement Systems Ltd, a spinout company from the University of Southampton (UK) and manufacturer of the MMS-14 CCFP Analyser. The rest of the authors declare no conflict of interest. 


\section{References}

ALPERIN, N. J., LEE, S. H., LOTH, F., RAKSIN, P. B. \& LICHTOR, T. 2000. MR-Intracranial pressure (ICP): a method to measure intracranial elastance and pressure noninvasively by means of $M R$ imaging: baboon and human study. Radiology, 217, 877-85.

BENIGNUS, V. 1969. Estimation of the coherence spectrum and its confidence interval using the fast Fourier transform. IEEE Transactions on Audio and Electroacoustics, 17, 145-150.

CAMPBELL-BELL, C. M., BIRCH, A. A., VIGNALI, D., BULTERS, D. O. \& MARCHBANKS, R. J. 2017. Reference intervals for the evoked tympanic membrane displacement measurement: a noninvasive measure of intracranial pressure. Physiol Meas.

CARLBORG, B., DENSERT, B. \& DENSERT, O. 1982. Functional patency of the cochlear aqueduct. Ann Otol Rhinol Laryngol, 91, 209-15.

CZOSNYKA, M., BRADY, K., REINHARD, M., SMIELEWSKI, P. \& STEINER, L. A. 2009. Monitoring of cerebrovascular autoregulation: facts, myths, and missing links. Neurocrit Care, 10, 373-86.

CZOSNYKA, M. \& PICKARD, J. 2004. Monitoring and interpretation of intracranial pressure. Journal of Neurology, Neurosurgery, and Psychiatry, 75, 813-821.

DUNN, L. T. 2002. Raised intracranial pressure. J Neurol Neurosurg Psychiatry, 73 Suppl 1, i23-7.

EIDE, P. K. \& KERTY, E. 2011. Static and pulsatile intracranial pressure in idiopathic intracranial hypertension. Clin Neurol Neurosurg, 113, 123-8.

FAES, L., PINNA, G. D., PORTA, A., MAESTRI, R. \& NOLLO, G. 2004. Surrogate data analysis for assessing the significance of the coherence function. IEEE Transactions on Biomedical Engineering, 51, 1156-1166.

FINCH, L. C., MARCHBANKS, R. J., BULTERS, D. \& BIRCH, A. A. 2018. Refining non-invasive techniques to measure intracranial pressure: comparing evoked and spontaneous tympanic membrane displacements. Physiol Meas, 39, 025007.

GHAJAR, J. 1995. Intracranial pressure monitoring techniques. New Horiz, 3, 395-9.

GOPEN, Q., ROSOWSKI, J. J. \& MERCHANT, S. N. 1997. Anatomy of the normal human cochlear aqueduct with functional implications. Hearing Research, 107, 9.

GWER, S., KAZUNGU, M., CHENGO, E., OHUMA, E. O., IDRO, R., BIRCH, T., MARCHBANKS, R., KIRKHAM, F. J. \& NEWTON, C. R. 2015. Abnormal intra-aural pressure waves associated with death in African children with acute nontraumatic coma. Pediatr Res, 78, 38-43.

GWER, S., SHEWARD, V., BIRCH, A., MARCHBANKS, R., IDRO, R., NEWTON, C. R., KIRKHAM, F. J., LIN, J.-P. \& LIM, M. 2013. The tympanic membrane displacement analyser for monitoring intracranial pressure in children. Child's Nervous System, 29, 927-933.

KIRKMAN, M. A. \& SMITH, M. 2014. Intracranial pressure monitoring, cerebral perfusion pressure estimation, and ICP/CPP-guided therapy: a standard of care or optional extra after brain injury? Br J Anaesth, 112, 35-46.

KONRADSSON, K. S., NIELSEN, L. H., CARLBORG, B. I. \& BORGKVIST, B. 2000. Pressure transfer between intracranial and cochlear fluids in patients with Meniere's disease. Laryngoscope, $110,264-8$.

LEHRER, J. F., OGUNLUSI, A., KNUTSEN, J. \& MARCHBANKS, R. J. 2009. The value of transcranial cerebral sonography in diagnosing neurootological disorders. Int Tinnitus J, 15, 164-7.

LUNDBERG, N. 1960. Continuous recording and control of ventricular fluid pressure in neurosurgical practice. Acta Psychiatr Scand Suppl, 36, 1-193.

MARCHBANKS, R. J. 1989. Method and apparatus for measuring intracranial fluid pressure. US patent application 4,841,986.

MARCHBANKS, R. J. 1996. Hydromechanical interactions of the intracranial and intralabyrinthine fluids. In: ERNST, A., MARCHBANKS, R. J. \& SAMII, M. (eds.) Intracranial and Intralabyrinthine Fluids: Basic Aspects and Clinical Applications. Berlin: Springer.

MOKRI, B. 2001. The Monro-Kellie hypothesis: applications in CSF volume depletion. Neurology, 56, $1746-8$. 
MOMJIAN, S., CZOSNYKA, Z., CZOSNYKA, M. \& PICKARD, J. D. 2004. Link between vasogenic waves of intracranial pressure and cerebrospinal fluid outflow resistance in normal pressure hydrocephalus. British Journal of Neurosurgery. Carfax Publishing Company.

MOSS, S. M., MARCHBANKS, R. J. \& BURGE, D. M. 1991. Long-term assessment of intracranial pressure using the tympanic membrane displacement measurement technique. Journal of Pediatric Surgery, 27, 927-928.

PANERAI, R. B. 2008. Cerebral autoregulation: from models to clinical applications. Cardiovasc Eng, 8, 42-59.

PHILLIPS, A. J. \& MARCHBANKS, R. J. 1989. Effects of posture and age on tympanic membrane displacement measurements. Br J Audiol, 23, 279-84.

REID, A., MARCHBANKS, R. J., BURGE, D. M., MARTIN, A. M., BATEMAN, D. E., PICKARD, J. D. \& BRIGHTWELL, A. P. 1990. The relationship between intracranial pressure and tympanic membrane displacement. Br J Audiol, 24, 123-9.

SAMUEL, M., BURGE, D. M. \& MARCHBANKS, R. J. 1998. Tympanic membrane displacement testing in regular assessment of intracranial pressure in eight children with shunted hydrocephalus. J Neurosurg, 88, 983-95.

SCHOSER, B. G., RIEMENSCHNEIDER, N. \& HANSEN, H. C. 1999. The impact of raised intracranial pressure on cerebral venous hemodynamics: a prospective venous transcranial Doppler ultrasonography study. J Neurosurg, 91, 744-9.

SHIMBLES, S., DODD, C., BANISTER, K., MENDELOW, A. D. \& CHAMBERS, I. R. 2005. Clinical comparison of tympanic membrane displacement with invasive intracranial pressure measurements. Physiol Meas, 26, 1085-92.

SHULMAN, A., GOLDSTEIN, B. \& MARCHBANKS, R. J. 2012. The tympanic membrane displacement test and tinnitus: preliminary report on clinical observations, applications, and implications. Int Tinnitus J, 17, 80-93.

SPIEGELBERG, A., PREUß, M. \& KURTCUOGLU, V. 2016. B-waves revisited. Interdisciplinary Neurosurgery, 6, 13-17.

TRABOULSI, R. \& AVAN, P. 2007. Transmission of infrasonic pressure waves from cerebrospinal to intralabyrinthine fluids through the human cochlear aqueduct: Non-invasive measurements with otoacoustic emissions. Hear Res, 233, 30-9.

WAGSHUL, M. E., EIDE, P. K. \& MADSEN, J. R. 2011. The pulsating brain: A review of experimental and clinical studies of intracranial pulsatility. Fluids Barriers CNS, 8, 5.

ZHANG, X., MEDOW, J. E., ISKANDAR, B. J., WANG, F., SHOKOUEINEJAD, M., KOUEIK, J. \& WEBSTER, J. G. 2017. Invasive and noninvasive means of measuring intracranial pressure: a review. Physiol Meas, 38, R143-r182. 